\title{
Data-Based Internal Model Controller Design For A Class of Nonlinear Systems
}

\author{
Ankush Ganeshreddy Kalmukale and Min-Sen Chiu ${ }^{*}$ \\ Department of Chemical and Biomolecular Engineering \\ National University of Singapore, 119260, Singapore
}

\begin{abstract}
An Internal Model Control (IMC) design strategy is proposed for a class of nonlinear systems that can be described by data-based modeling technique called Just-in-Time Learning (JITL). The proposed controller consists of a conventional linear controller augmented by a series of correction terms to account for nonlinearities in the system. The resulting controller is shown to have superior performance when compared with a linear IMC controller. This is evaluated by a case study of a polymerization reactor.
\end{abstract}

Keywords: Nonlinear systems; Just-in-time-learning; Internal model control

\section{Introduction}

Although many processes exhibit significant nonlinear behavior, most controller design techniques are based on linear models. The prevalence of linear control strategies is primarily due to two reasons. First, there are well-established methods for the development of linear models from input-output data while practical identification techniques for nonlinear models are still being developed. Furthermore, controller design for nonlinear models is considerably more difficult than for linear models (Nahas et al., 1992). In available linear control strategies, linear IMC is a convenient and powerful controller design strategy for the open-loop stable dynamic systems (Morari and Zafiriou, 1989). This is mainly due to two reasons. First, integral action is included implicitly in the controller. Moreover, plant/model mismatch can be addressed via the design of a robustness filter. IMC design is expected to perform satisfactorily as long as the plant is operated in the vicinity of the point where the process model is obtained. However, many chemical processes exhibit a certain degree of nonlinearity. Furthermore, different operating conditions are usually necessitated by the external factors such as the persistent load disturbances or the increasingly demand of product diversification and cost reduction, e.g. grade changeover in a polymerization reactor. Under this situation, the process dynamics is forced away from its nominal design condition, which exacerbates the effect of the inherent nonlinear nature of the process. As a result, the performance of linear IMC controller will degrade or even become unstable. The extension of linear IMC strategy to nonlinear systems has been popular model-based control approach. Several nonlinear IMC schemes have been proposed in

* Author/s to whom correspondence should be addressed: checms@nus.edu.sg 
literature. Among these results, Doyle et al. (1995) has proposed a partitioned model inverse controller synthesis scheme based on Volterra model that retains the original spirit and characteristics of conventional (linear) IMC while extending its capabilities to nonlinear systems. When implemented as part of the control law, the nonlinear controller consists of a standard linear controller augmented by a nonlinear correction term. Furthermore, the nonlinear correction term can be "turned off" on-line, with the control law reverting to the chosen linear control scheme. It is this flexibility that gives partitioned model inverses great promise in nonlinear control schemes. However, Volterra model derived using local expansion results such as Carleman linearization is accurate for capturing local nonlinearities around an operating point, but may be erroneous in describing global nonlinear behavior (Doyle et al., 1995). Another drawback is that second-order Volterra models require many parameters to describe nonlinearities.

Harris and Palazoglu (1998) has proposed similar control scheme as Doyle et al. (1995) by using functional expansion (FEx) models instead of Volterra model. However, these models are limited to fading memory systems and the radius of convergence is not guaranteed for all input magnitudes. Maksumov et al. (2002) used neural network (NN) as a nonlinear model and a linear ARX model in partitioned model inverse controller design in an experimental study of a pH neutralization process. Inspired by the previous work, a nonlinear IMC design strategy is proposed for a class of nonlinear systems that can be modelled by data-based modeling technique called Just-In-Time Learning (JITL). JITL has been shown to have better modeling accuracy over the traditional black-box modeling technique like NN (Bontempi et al., 2001). In section 2, the proposed control strategy is discussed in detail and a polymerization reactor example is used to illustrate the proposed controller design method in section 3 .

\section{Proposed Control Strategy}

\subsection{Just-in-Time Learning (JITL)}

There are three main steps in JITL to predict the model output corresponding to the query data: (1) relevant data samples in the database are searched to match the query data by some nearest neighborhood criterion; (2) a local model is built based on the relevant data; (3) model output is calculated based on the local model and the current query data. The local model is then discarded right after the answer is obtained. When the next query data comes, a new local model will be built based on the aforementioned procedure (Bontempi et al., 2001).

In the literature, distance measures are overwhelmingly used in the JITL to evaluate similarity between two data samples. Recently, Cheng and Chiu (2004) developed an enhanced JITL methodology by exploring both distance measure and the complementary information available from the angular relationship. In this work, we have incorporated this enhanced JITL methodology as a modeling tool in the proposed control structure. The detail algorithm is referred to Cheng and Chiu (2004).

\subsection{Nonlinear IMC strategy}

The IMC structure (Morari and Zafiriou, 1989) has enjoyed considerable success for linear control system design and analysis. The general IMC structure is illustrated in 
Fig. 1, where $P$ is the process to be controlled, $M$ represents the model of the process, and $Q$ is the IMC controller.

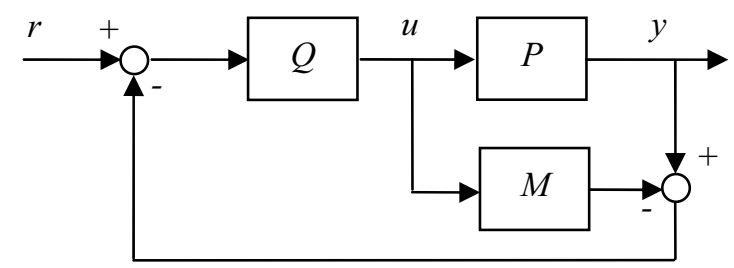

Figure 1. General IMC structure

This structure is sufficiently general to allow the use of variety of process models, such as fundamental nonlinear models, as well as $\mathrm{NN}$ and black-box-type models. The difficulty in the use of these models in the IMC strategy arises in the design of IMC controller. Because the controller is based on the inverse of the model $M$, a reliable and efficient method is required to achieve this inversion (Maksumov et al., 2002). In literature, numerical and analytical inversion techniques have been employed; however these approaches can be computationally demanding.

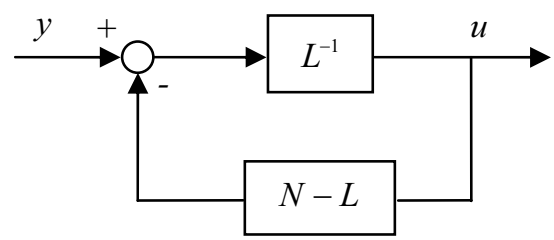

Figure 2. Partitioned model inverse

In this work, we utilize a partitioned model to yield a flexible nonlinear model inversion (Maksumov et al., 2002). Considering a process for which a linear $(L)$ and a nonlinear $(N)$ model are available, the models can be combined into a composite model $M$ as

$M=L+(N-L)$

The inverse can then be determined as

$M^{-1}=\left[I+L^{-1}(N-L)\right]^{-1} L^{-1}$

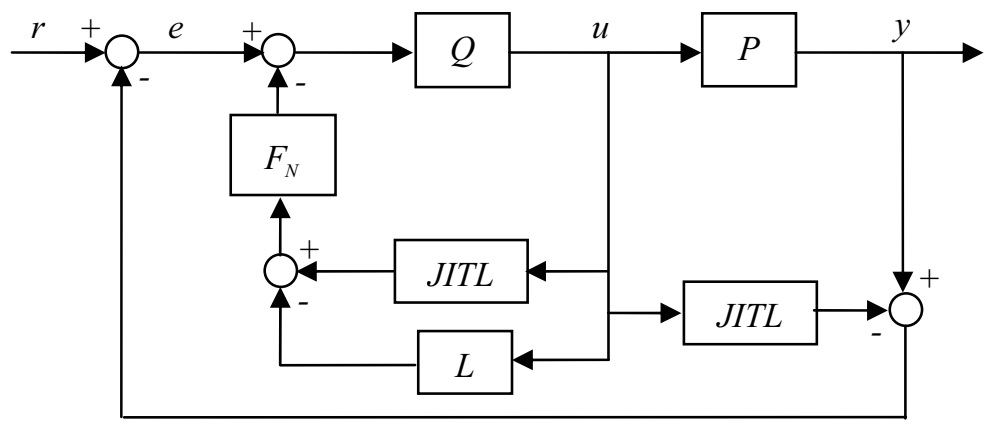

Figure 3. IMC structure with partitioned controller 
Note that only the inverse of the linear model is required. Additionally, this inverse can be computed on-line using the feedback loop illustrated in Fig. 2. Here, we use this partitioned model inverse structure in IMC control scheme, with linear model $L$ obtained around an operating point and nonlinear model obtained by JITL algorithm. The resulting IMC controller, referred to as nonlinear IMC (NLIMC) henceforth, has the structure illustrated in Fig. 3, where $Q$ is the standard IMC controller

$Q=L_{-}^{-1} F_{L}$

where $L_{-}$is the minimum phase of linear model and $F_{L}$ is a low-pass filter. Typically, this filter is given by

$F_{L}(s)=\frac{1}{(\alpha s+1)^{r}}$

where $r$ is the relative degree of the system and $\alpha$ acts as a tuning parameter.

The second filter $F_{N}$ is used to provide robustness for the nonlinear IMC. $F_{N}$ would ideally be chosen as the inverse of $F_{L}$; however, this choice for second filter amplifies noise in the controller's feedback loop. A more practical choice for this filter is given by (Harris and Palazoglu, 1998)

$F_{N}(s)=\frac{F_{L}^{-1}(s)}{(\beta s+1)^{r}}$

where $\beta$ is a tuning parameter and typically $\beta<\alpha$.

\section{Case Study}

In this section we consider the application of NLIMC to a polymerization reaction taking place in a jacketed CSTR. The reaction involves free-radical polymerization of methyl methacrylate (MMA) with azo-bis-isobutyronitrile (AIBN) as initiator and toluene as solvent. The model is represented by the following equations (Doyle et al., 1995):

$\frac{d C_{m}}{d t}=-\left(k_{p}+k_{f_{m}}\right) C_{m} P_{0}+\frac{F\left(C_{m_{i n}}-C_{m}\right)}{V}$,

$\frac{d C_{I}}{d t}=-k_{I} C_{I}+\frac{F_{I} C_{I_{i n}}-F C_{I}}{V}$,

$\frac{d D_{0}}{d t}=\left(0.5 k_{T_{c}}+k_{T_{d}}\right) P_{0}^{2}+k_{f_{m}} C_{m} P_{0}-\frac{F D_{0}}{V}$,

$\frac{d D_{I}}{d t}=M_{m}\left(k_{P}+k_{f_{m}}\right) C_{m} P_{0}-\frac{F D_{I}}{V}$,

where $P_{0}=\left[\frac{2 f^{*} k_{I} C_{I}}{k_{T_{d}}+k_{T_{c}}}\right]^{1 / 2}$. 
Owing to the space constraint, the physical parameters of this reactor are not included and readers can refer to Doyle et al. (1995) for the details. The control problem focuses on manipulating the volumetric flowrate of the initiator $\left(u=F_{I}\right)$ in order to regulate the number-average molecular weight $\left(y=D_{I} / D_{0}\right)$. The nominal operating condition chosen for this study is $u_{0}=0.016783$ and $y_{0}=25000.5$. From the first-principles reactor model, a linear model was obtained around this operating point by Taylor series approximation. The inverse of this linear model is augmented by a linear filter to obtain the linear IMC controller $(Q)$ with tuning parameters $r=2, \alpha=0.5$. NLIMC consists of the identical $Q$ and the second filter $F_{N}$ is designed with $\beta=0.1$. Fig. 4 shows the response of two controllers for $\pm 50 \%$ step changes in setpoint. It can be seen that NLIMC tracks reference trajectory much better than linear IMC. Reference trajectory is the linear filter response, which represents ideal system response in case of IMC structure when the process model is perfect. To evaluate disturbance rejection capability of the proposed control strategy, unmeasured $\pm 25 \%$ step disturbances in inlet initiator concentration are assumed and the simulation results are displayed in Fig. 5. It is evident that control performance using NLIMC scheme is better than linear IMC. In both cases considered above, NLIMC scheme has reduced the integral square tracking error, relative to linear IMC scheme, by a margin of approximately $80-95 \%$.
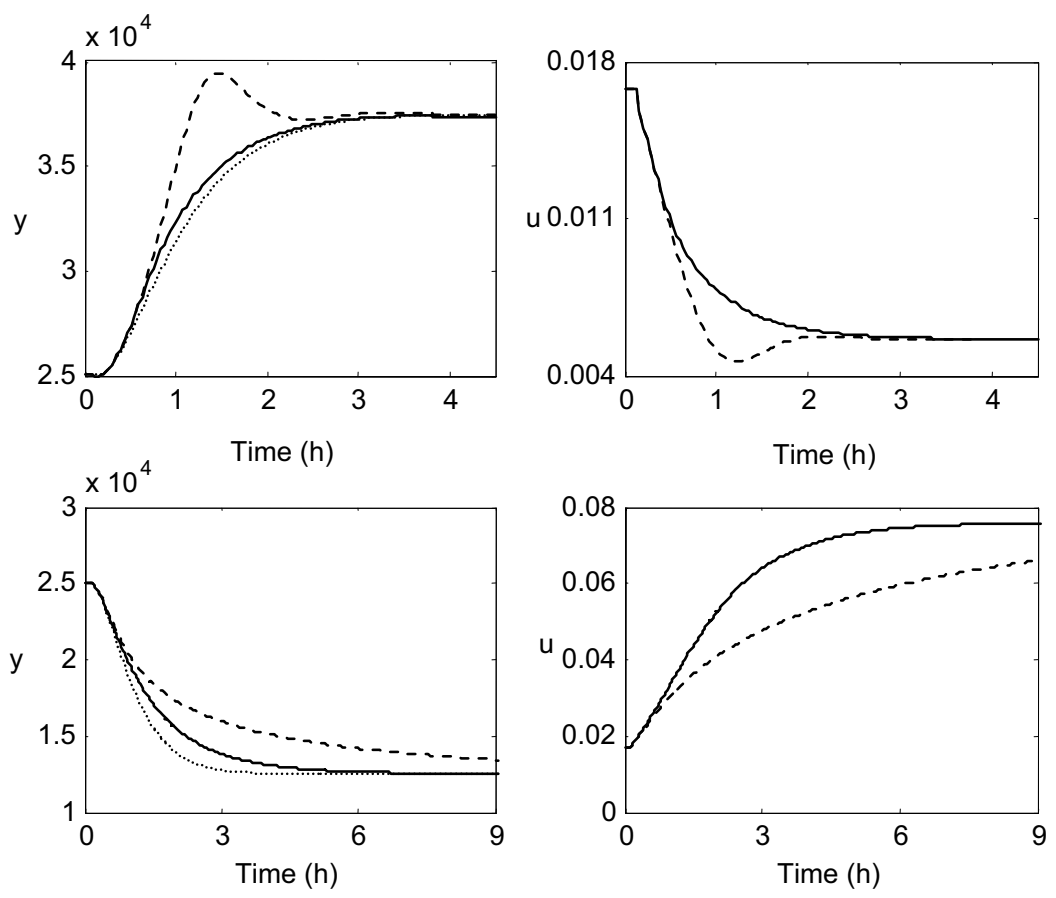

Figure 4. Closed-loop responses for $\pm 50 \%$ step changes in the setpoint. Dotted: reference; dash: linear IMC; solid: NLIMC. 

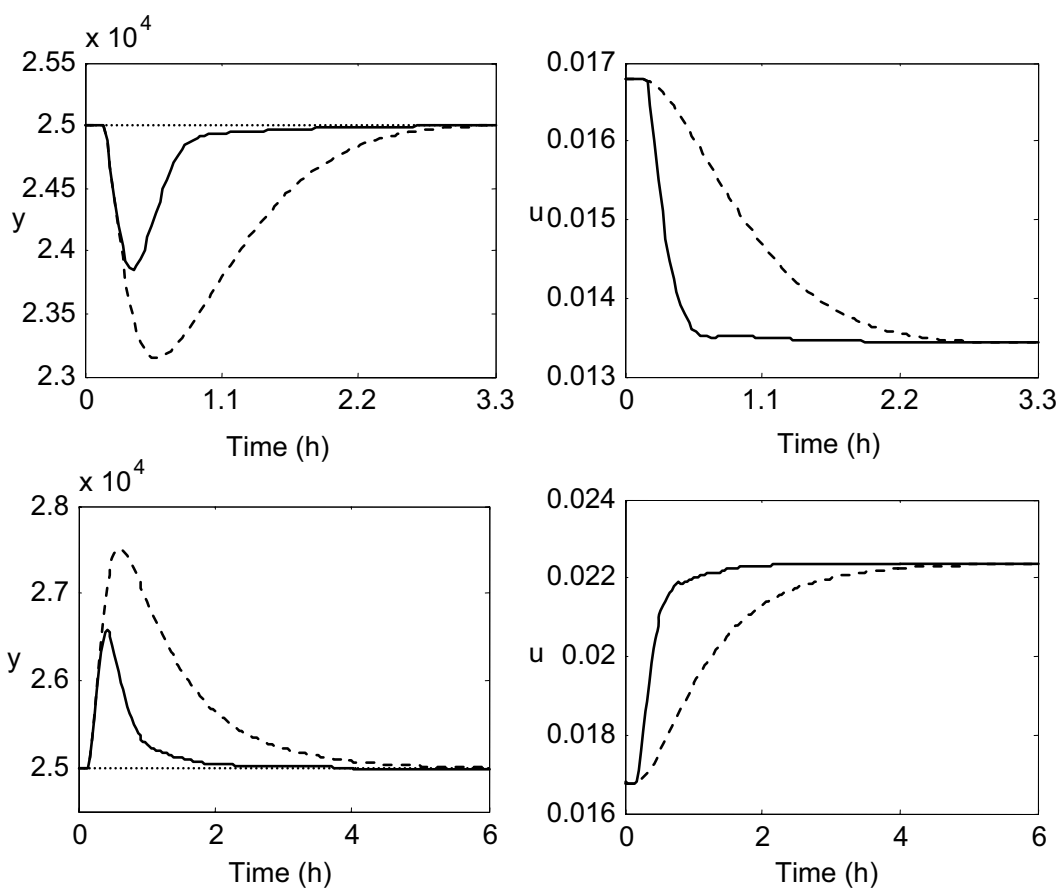

Figure 5. Closed-loop responses for $\pm 25 \%$ step disturbances in inlet initiator concentration $\left(C_{I_{i n}}\right)$.Dotted: reference; dash: linear IMC; solid: NLIMC.

\section{Conclusion}

An internal model control strategy utilizing a partitioned model is proposed for nonlinear systems using JITL as an estimator of process dynamics. The fact that only a linear inversion is required in the synthesis of this controller is an attractive feature of this control scheme. Simulation results for a case study of polymerization reactor indicate that the proposed control scheme is able to provide better performance over a large range of operation as compare to a linear IMC.

\section{References}

Bontempi, G., H. Bersini and M. Birattari, 2001, The local paradigm for modeling and control: from neuro-fuzzy to lazy learning. Fuzzy Sets and Systems 121, 59-72.

Cheng, C. and M.S. Chiu, 2004, A new data-based methodology for nonlinear process modeling. Chemical Engineering Science 59, 2801-2810.

Doyle, F.J., B.A. Ogunnaike and R.K. Pearson, 1995, Nonlinear model-based control using second-order Volterra models. Automatica 31, 697-714.

Harris, K.R. and A. Palazoglu, 1998, Studies on the analysis of nonlinear process via functional expansions-III: Controller design. Chemical Engineering Science 53, 4005-4022.

Maksumov, A., D.J. Mulder, K.R. Harris and A. Palazoglu, 2002, Experimental application of partitioned model-based control to $\mathrm{pH}$ neutralization. Ind. Eng. Chem. Res. 41, 744-750.

Morari, M. and E. Zafiriou, 1989, Robust Process Control. Prentice-Hall, Englewood Cliffs, NJ.

Nahas, E.P., M.A. Henson and D.E. Seborg, 1992, Nonlinear internal model control strategy for neural network models. Computers Chem. Engng. 16, 1039-1057. 